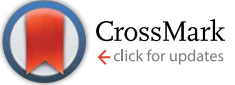

Cite this: RSC Adv., 2017, 7, 3089
Received 24th October 2016 Accepted 22nd December 2016

DOI: 10.1039/c6ra25765h

www.rsc.org/advances

\section{Screening for the bioactive constituents of traditional Chinese medicines-progress and challenges}

\author{
Xing-Xin Yang, ${ }^{a}$ Wen Gu, ${ }^{a}$ Li Liang, ${ }^{a}$ Hong-Li Yan, ${ }^{a}$ Yan-Fang Wang, ${ }^{a}$ Qian $\mathrm{Bi}^{,}{ }^{a}$ \\ Ting Zhang, ${ }^{a}$ Jie $\mathrm{Yu}^{* a b}$ and Gao-Xiong Rao*ab
}

\begin{abstract}
The search for lead compounds from traditional Chinese medicines (TCMs) may be promising for new drug development. Screening efficiency and efficacy is chiefly determined by the validity of the selected screening tools. Recently, many experimental screening tools have been developed to identify lead compounds. This review provides a toolbox of the experimental screening methods and models that have been applied to discover new lead compounds and drugs from TCMs. The merits and limitations of these tools are summarized, assisting readers in the rapid retrieval of useful information and providing reference values for selecting appropriate screening tools for different research.
\end{abstract}

\section{Introduction}

Traditional Chinese Medicines (TCMs) count among the world's most ancient herbal medicines and have been used extensively by TCM practitioners for thousands of years. ${ }^{1}$ TCM has played an indispensable role in the prevention and treatment of human disease, ${ }^{\mathbf{1 - 3}}$ especially in complicated and chronic diseases such as rheumatism and arthritis. As a complementary therapeutic technique with fewer side effects than western medicines, TCM is attracting increasing attention. The chemical synthesis of new drugs has rapidly developed in recent years with the advancement of combinatorial chemistry and computer-aided drug design technology. However, the exploration of drugs and lead compounds from TCMs remains a significant avenue for drug development, ${ }^{4-6}$ because of the novel structures, therapeutic abilities, and certain unique pharmacological effects of the chemical substances in TCMs. The screening of bioactive constituents from TCMs is of great significance in innovative drug discovery with medicinal herbs as lead compounds.

The clarification of the active constituents of TCMs is a precondition to understanding their action mechanism, and provides the foundation and core of TCM quality control. It also guards the safety, effectiveness, stability, and controllability of TCMs. However, multiple substances in TCMs and their likely complex mechanisms of action complicate screening and

${ }^{a}$ College of Pharmaceutical Science, Yunnan University of Traditional Chinese Medicine, 1076 Yuhua Road, Kunming 650500, Yunnan Province, P. R. China. E-mail: cz.yujie@gmail.com; rao13987124569@qq.com; Fax: +86-871-65933303; +86-871-65918232; Tel: +86-871-65933303; +86-871-65918232

${ }^{b}$ Engineering Laboratory for National Healthcare Theories and Products of Yunnan Province, 1076 Yuhua Road, Kunming 650500, Yunnan Province, P. R. China analysis for bioactive constituents, even from well-documented formulations for specific diseases. This acutely restrains the development and modernization of TCM. Thus, the development of screening methods and models that efficiently search for bioactive constituents within the complex matrices of TCMs is important for elucidating the pharmacodynamic essence (including effective substances and action mechanisms) of TCMs and in the development of new drugs from them.

A variety of screening methods and models (Fig. 1) have been developed and used to screen for the bioactive constituents of TCMs. ${ }^{6-8}$ These include animal, tissue/organ, cell, and receptor/ enzyme models; serum pharmacology; biochromatography; gene chip technology; molecularly imprinted polymers and spectrum-effect relationships. Although widely applied, these screening tools may cause confusion, with difficulty in selecting suitable ones, due to their considerable diversity. In this review, these screening tools are identified, and their merits and limitations are summarized simply yet comprehensively. This may assist readers in the rapid retrieval of useful information for the selection and application of suitable screening tools for different research objectives.

\section{In vivo screening methods and models}

\subsection{Animal models}

The extraction of a single compound or single distilled fraction from natural products and the evaluation of its bioactivity in classic pathological animal models is a routinely used screening process. Rat, mouse, rabbit, beagle dog, monkey, and zebra fish are commonly used model animals. The animal model is the most classic pharmacological screening model, and is 


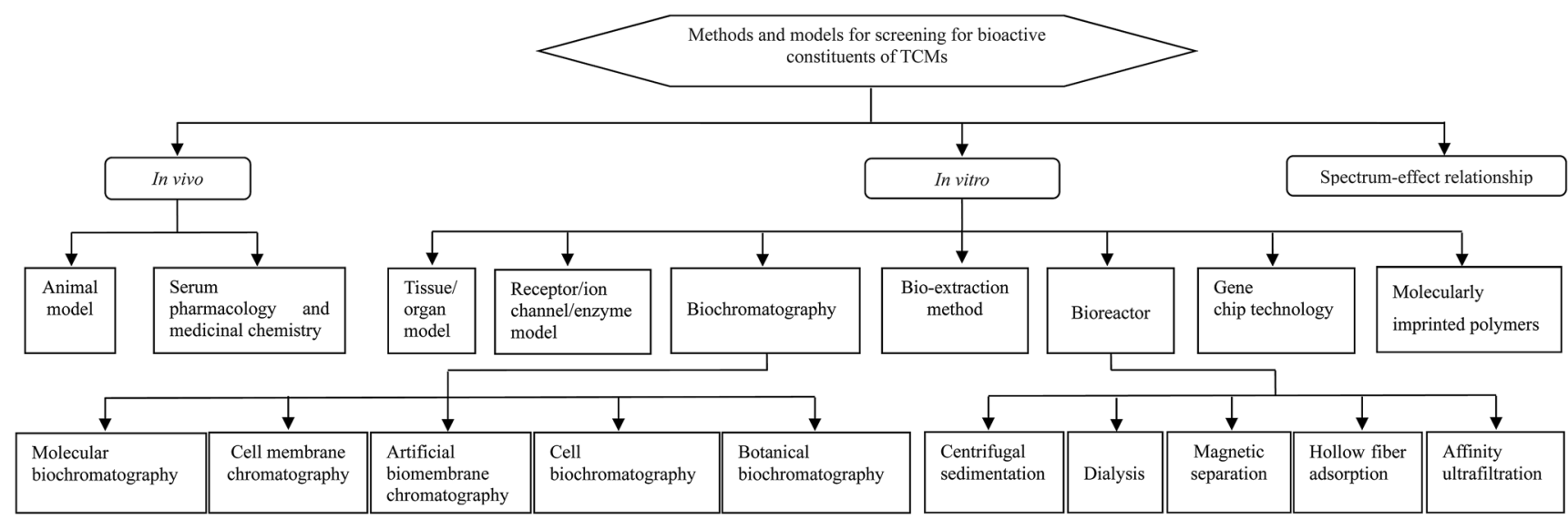

Fig. 1 Methods and models currently used to screen for the bioactive constituents in TCMs.

important in medicine evaluation as it can mimic the physiopathological and clinical features of patients, demonstrating the efficacy, side effects, and toxicity of medicines. Although the use of animal models is laborious and time-consuming, and their screening throughput is low, it remains the primary indispensable method of drug discovery and evaluation. ${ }^{6,8}$

\subsection{Serum pharmacology and serum medicinal chemistry}

Although TCMs contain numerous complex chemical substances, detection of distinct compounds in TCMs after they enter the bloodstream are extremely limited. ${ }^{8}$ TCM ingredients that enter the bloodstream comprise the "crude extract" of serum, which includes constituents present in TCMs and formed in the process of decoction, as well as metabolites and endogenous bioactive substances in the body generated by stimulation from TCMs. ${ }^{9}$ The effectiveness of serum containing crude extract is evaluated using serum pharmacology, whereas serum medicinal chemistry is the process of isolating and identifying the active compounds in serum. The analytical process begins with the intragastric administration of TCM extracts. The serum is then separated and its bioactivity evaluated by pharmacological means, in vitro and in vivo. Finally, the bioactive constituents in the serum are separated and identified. ${ }^{8}$ As such, the chemical substances of TCMs that exert certain pharmacologic actions may be revealed. Substance effectiveness of many types of TCMs has been researched using this method, with valuable results obtained..$^{8-11}$

This method has the following advantages: (1) interference from the physical and chemical properties of crude TCM preparations is excluded, such as $\mathrm{pH}$, osmotic pressure, and the presence of electrolytes or tannins; (2) TCM ingredients in the gastrointestinal tract that cannot enter the bloodstream are excluded, which simplifies exploration; (3) serum from the administered animal will contain the authentic effective TCM substances; (4) the influence of many factors that affect in vitro experiments is avoided, potentially reflecting the effectiveness of TCMs more accurately and authentically.

However, there are also some deficiencies in this method: (1) the separation of bioactive constituents from complex biological samples (serum) is more difficult than direct separation from crude TCM extracts; (2) the inference of information about the structure of bioactive compounds in TCMs from their metabolites is difficult; (3) some compounds detected in the serum may be inactive ingredients in TCMs; (4) the efficacy of purified compounds that appear in serum is only evident when the blood concentration of the compound exceeds the original concentration; (5) operational protocols require standardization for dose schedule, time and method of blood collection, serum pretreatment, differences in drug absorption from individual animals, and differences in the composition of serum and the concentration of compounds from dosage and sampling time. Therefore, although the application of this method has excellent prospects, problems remain that need to be addressed further.

\section{In vitro screening methods and models}

\subsection{Tissue/organ models}

With the development of modern medicine and pharmacology, a growing number of animal tissue/organ models have been established for evaluating the activity of drugs, such as the isolated vascular ring, heart/liver perfusion and tissue culture models. By investigating the effects of a drug on specific tissues or organs, possible pharmacological activity and mechanism of action may be explored. This model has more recently become an important means for activity screening. It overcomes some of the deficiencies of the animal model in the following ways: ${ }^{8}(1)$ the amount of screening sample required is less. Generally, a sample of between 1-5 $\mathrm{g}$ or more is required for use in an animal model (depending on the dosage and the size of the animal used), while the sample amount required in a tissue/ organ model is as low as or less than one-tenth of that used in an animal model; (2) labor intensity and animal consumption are less, and the screening scale may be expanded; in some models only a small amount of tissue or organ (such as the isolated vascular ring model) is required for a complete screening. Multi-sample screening can be performed 
simultaneously, improving efficiency and decreasing costs; (3) the interference from other in vivo factors is reduced, allowing more precise evaluation.

Despite the advantages of screening using tissue/organ models, limitations exist, such as a small screening scale, low efficiency, limited pharmacological indices and difficulty of realizing high-throughput and high-content screening. The more recent application of tissue microarray technology ${ }^{\mathbf{1 2 , 1 3}}$ is improving screening efficiency and drug target identification at the tissue/organ level, with advantages of high throughput, low experimental error and abundant information capacity. Tissue microarray technology is also time-saving, and small sample volumes can be handled.

\subsection{Cell model}

The cell model is the most widely-used drug screening model, and not only more closely mimics the pathophysiological state of various diseases, but also provides useful information about the predictability of bioactive compounds in vivo, as well as on permeability and toxicity. It shows considerable advantage over whole-animal and tissue/organ models: (1) screening efficiency is greatly improved, due to the simple and fast operation of cell experiments, the short time period for cell growth, and the applicability of large-scale cultures; (2) factors interfering with efficacy are reduced and easier to control, making screening results more stable and reliable; (3) high throughput screening can be achieved as many compounds can be screened simultaneously. ${ }^{\mathbf{1 4 , 1 5}}$ This makes it possible to find drugs with excellent activity solely by expanding the screening objects and scope; (4) due to the low-consumption sample amounts used in a cell model, a small amount of isolated compound (fraction) can be used with multiple pathological models, improving the probability of discovering new drugs and lead compounds; (5) high content screening can be performed. Because the action of a drug on cells may be demonstrated in a variety of ways, its efficacy or toxicity is more thoroughly revealed with a cell model, by expanding the detection range and concurrently monitoring multiple pharmacological indices. ${ }^{16,17}$

Nevertheless, in vitro cell models cannot mimic the dynamic procedures of drugs in the human body, and do not exactly reflect pharmacological behavior of drugs in vivo. The efficacy of some active compounds identified by cell models may be reduced or absent once in the circulation. Thus, cell model screening processes should simulate the body's internal environment as far as possible. Additionally, activity screening using cell models remains a process in which the effect of samples on cells is observed; this cannot reveal specific pathways and targets of drug action, and only shows comprehensive effects of the drug on cell growth processes.

\subsection{Receptor/ion channel/enzyme model}

With the development of enzymology and receptor theory, drug targets have been revealed and new drug targets discovered consecutively. So far, more than 600 types of drug target have been found. The use of receptors, ion channels or enzymes as targets to search for bioactive constituents is an important method in new drug discovery. ${ }^{\mathbf{8 1 8 , 1 9}}$ Within this process, the receptor, enzyme or channel is stimulated by the tested compound, and a variety of pharmacological indices are then detected and binding intensity between the compound and target is evaluated. This model has a number of characteristics: (1) the screening process is cut short, with high-detection sensitivity and potent specificity, enabling high-throughput and high content screenings; (2) screening occurs at the binding site, and not only is binding information between drug and target obtained, but the activity of the drug can be understood and functional screening performed; (3) some limitations of an animal model are overcome. For example, diseases can be screened for if an appropriate animal model is not available. Moreover, some drugs are metabolized in the animal gut or liver and may not arrive at their targets, diminishing the ability to assess their activity in a whole animal model.

Major TCM compounds are not highly bioactive; compounds do not bind tightly with targets in this model resulting in low screening efficiency. In addition, screening results do not completely reflect multi-target and multi-pathway characteristics of effective TCM substances. Active compounds that bind with targets in this model may not provide the expected pharmacological effect after entering the body and, therefore, may not be developed as a drug.

\subsection{Biochromatography}

A variety of bioactive materials (enzymes, carrier proteins and cell membranes) or biomimetic membrane materials (liposomes or artificial cell membranes) are used in novel affinity chromatography method known as biochromatography, when a stationary phase is used. Because biological activity occurs with a stationary phase and various bioactive substances are specifically bonded, biochromatography has been recently applied to the screening, separation, and preparation of active substances from mixtures. ${ }^{\mathbf{2 0 , 2 1}}$ Biomacromolecules, cell membrane (or biomimetic membrane), living cells and plant cells (or cell walls) have been fixed on a chromatographic carrier (e.g. silica and gel) to form different biochromatography stationary phases. Biochromatography may be subdivided into molecular biochromatography, cell membrane chromatography (or artificial biomembrane chromatography), cell biochromatography, and botanical biochromatography, according to the chromatographic stationary phase used.

These methods have the characteristics of both chromatographic separation and biological activity, and separation and recognition of biological activity can be achieved simultaneously while screening for bioactive constituents. Therefore, active constituents not only bind and are selectively recognized during the stationary phase, but interference from inactive ingredients in a complex matrix such as that found in TCMs is directly eliminated, contributing to an efficient screening when used with TCMs. ${ }^{20,22}$ Two-dimensional "recognition-separation-identification" chromatographic systems have been developed by combining biochromatography with liquid chromatography/mass spectrometry (LC/MS) or gas chromatography/mass spectrometry (GC/MS), enabling highly 
efficient screening for bioactive constituents in complex TCM samples. ${ }^{23-29}$

Biochromatography technology has been widely used for TCM research, but disadvantages remain: (1) the bioactivity of a stationary phase can only be preserved for a short time, which may lead to failure of commercialization of the chromatographic column; (2) the pressure and temperature requirements of columns, and the properties and flow rate of a mobile phase, are highly specific. A chromatographic environment with high pressure and anoxia is not amenable to the maintenance of bioactivity in a stationary phase in the analytical process; (3) the preparation procedure of a stationary phase is complicated. A stationary phase with bioactivity is easy to inactivate allosterically during preparation and use. Furthermore, stationary phases prepared in different laboratories and used in different environments produce different results, challenging the reproducibility and applicability of biochromatography; (4) the amount of active constituents specifically retained by the stationary phase is usually small, making it difficult to obtain sufficient sample quantities for the identification of molecular structure or pharmacological verification trials. In summary, further improvements to the performance and dependability of biochromatography technology are required before widespread application of this technique to screening of TCMs.

3.4.1. Molecular biochromatography. Active biomacromolecules, such as enzymes, receptors, antibodies, transmission proteins, and DNA, immobilized on chromatographic carriers, usually serve as the stationary phase of molecular biochromatography. The different ingredients may interact within the stationary phase with different binding intensity when they are moved in the column with the mobile phase, which has different retention features on the column. The screening and separation of bioactive constituents in TCMs can be performed using this discrepancy of chromatographic retention, and binding variables (such as plasma-protein binding rate and ligand-receptor affinity) between active compound and target can also be determined. New active compounds and their targets can be identified, and their mechanism of action can be better understood..$^{20,21,30}$

This technology has been applied widely and Wang et al. ${ }^{31}$ used a chromatographic column filled with stationary phase of silica-bonded human serum albumin to screen Angelica sinensis extract, confirming a finding of the two most effective compounds previously reported in the literature. Additionally, Wang et al. ${ }^{26}$ used a stationary phase of silica-bonded human serum albumin with LC/MS to screen and analyze an extract of Longdan Xiegan decoction. They found hundreds of compounds interacting with human serum albumin, of which 19 were identified using LC/MS. Su et al..$^{32}$ applied a stationary phase of silica-bonded DNA to screen for TCM extracts. The chromatographic retention time of the screened compounds correlated well with their affinity for DNA, and multiple active compounds that could bind to DNA were identified from these complex TCM samples. Min et al..$^{33}$ coated acetylcholinesterase (AChE) on a capillary wall to prepare a bioactive stationary phase of capillary electrophoresis. The prepared phase was then used to screen for a variety of TCM extracts, including 30 extracts that interact with AChE, so this method may be used to screen for AChE inhibitors.

3.4.2. Cell membrane chromatography. Cell membrane chromatography (CMC) $)^{22,34,35}$ is a bioaffinity chromatography technique that combines biological interactions with chromatographic separation. CMC uses a cell membrane stationary phase (CMSP), which is prepared by fixing active cell membranes from tissue or cultured cells on the surface of a support such as silica. Cell membranes contain embedded receptors, ion channels, and enzymes, which participate in cell signaling for communication with surrounding cells and tissues, maintenance of cellular homeostasis, and cellular activity. With CMSP, chromatographic separation and the recognition of biological activity can be carried out simultaneously. Active constituents in TCMs are selectively retained by the chosen CMSP, which reduces interference from impurities. CMC offers a high level of performance, selectivity, specificity, and efficiency for analyzing and screening active compounds from TCMs. Therefore, it is particularly well suited to screening for bioactive compounds in TCMs and natural product libraries.

A variety of CMC models have been developed using cell membranes from different tissues (vascular smooth muscle, myocardium, and cerebral vessel) or cultured cell lines (red blood cells, macrophages, and cells with high expression of CD40, EGFR, and $\alpha 1 \mathrm{AAR}$ ). By combining online (or offline) with LC/MS or GC/MS, these CMC models have been widely applied to the screening of bioactive constituents from TCM extracts, and data show that CMC can be used to screen for active constituents of TCMs. ${ }^{22,34,35}$

3.4.3. Artificial biomembrane chromatography. Artificial biomembrane chromatography ${ }^{20,36}$ is affinity chromatography with an artificial biomembrane stationary phase prepared by fixing liposomes, egg lecithin, or soybean lecithin on a chromatographic carrier such as silica. This method can be applied to study the process of membrane permeation by a drug, using chromatographic principles. Because the artificial biomembrane stationary phase simulates the lipid bilayer structure of cell membrane, it not only can be used to study how drugs permeate the membrane but can also be applied to predict the activity parameters of drugs and separate enzymes or proteins from mixtures. Moreover, certain ligands can be embedded in the artificial biomembrane for specific chromatographic analysis. Zhang et al. ${ }^{37}$ used a monolithic column filled with artificial biomembrane stationary phase prepared with liposomes, to screen three TCM extracts (Glycyrrhizae Radix, Scutellariae baicalensis Radix and Sophorae Flos), which identified several active compounds that could penetrate the biomembrane. Wang et al. ${ }^{27}$ developed a comprehensive twodimensional chromatographic system by combining a monolithic column filled with an artificial biomembrane stationary phase of liposomes with LC/MS, to screen Schisandra chinensis extract; 14 active compounds were found to interact with the artificial biomembrane stationary phase. Wang et al. ${ }^{28}$ discovered and identified 10 active compounds (including 8 flavonoids and 2 iridoid glycosides) from Longdan Xiegan decoction that could penetrate liposome biomembranes, using a comprehensive two-dimensional chromatographic system that 
combined a monolithic column of liposome biomembrane with LC/MS. These results suggest that artificial biomembrane chromatography is applicable for screening active constituents in TCMs that can penetrate membranes.

3.4.4. Cell biochromatography. With cell biochromatography, human or animal living cells are immobilized on a chromatographic carrier such as gel to fabricate a bioactive stationary phase, and the interaction between drugs and living cells is assayed with chromatographic principles and techniques. Zeng et al. ${ }^{38}$ fixed human red blood cells on a gel carrier to form a biochromatographic stationary phase, and used this stationary phase to distinguish 2 stereoisomers of glucose, exploiting the divisional ability of glucose transport protein (Glut 1) in the membrane of red blood cells in the stationary phase. Although activity can be recognized simultaneously with chromatographic separation, it has not yet been used to screen active constituents from TCM extracts.

3.4.5. Botanical biochromatography. With botanical biochromatography, plant cells or cell walls are immobilized on a chromatographic carrier such as gel to form a stationary phase. This phase is performed for the enrichment and purification of target compounds in mixtures. Ehwald et al. ${ }^{\mathbf{3 9 , 4 0}}$ prepared a biological stationary phase using cell walls from a resuspended culture solution of Chenopodium album L., and applied the phase to separate biomacromolecules. At this time, this technique has not been applied to screen TCMs for active constituents.

\subsection{Bio-extraction method}

With bio-extraction, the analyzed sample is incubated with bioactive material (such as enzymes, receptors, carrier proteins, cell membranes, DNA, live cells, and liposomes), to bind active constituents with active material. The bound active constituents are then separated from complex matrices using specific separation techniques. As with biochromatography, bio-extraction can be used to identify biological activity and for chromatographic separation. The active constituents of complex samples such as TCMs can be recognized and separated, with direct elimination of interference from inactive ingredients.

Unlike biochromatography, the screening target for bioextraction does not require immobilization on a chromatography carrier, thus avoiding the complicated stationary phase preparation and packing process of a column, which is prone to maintaining the stereoisomers and biological activity of the screening target. Combined with online or offline LC/MS or GC/ MS, bio-extraction can be performed for the convenient identification and analysis of active constituents in TCMs and has recently received widespread attention and application in TCM research. Several separation techniques may be used, including centrifugal sedimentation, dialysis, magnetic separation, hollow fiber adsorption, and affinity ultrafiltration, with a suitable separation technique selected according to screening target and sample characteristics.

3.5.1. Centrifugal sedimentation. With centrifugal sedimentation, screening targets (such as cells or cell membranes) that bind with active constituents are precipitated by centrifugation, and then the supernatant containing inactive ingredients is directly removed. The bound active constituents are then dissociated by damaging the precipitated targets with organic solvent, and the damaged targets are precipitated by centrifugation. Remaining supernatant containing active constituents is then analyzed. Advantages of this technique include a concise operational process and low cost of analysis. However, some inactive ingredients are not easily removed by centrifugal sedimentation (inactive ingredients are easily precipitated with the sedimentation of screening targets). Furthermore, some bound active ingredients are easily dissociated and cannot be determined during the impurity washing process. Additionally, the method is unsuitable for screening for trace quantities of active compounds, and centrifugal sedimentation is limited to screening cell- and cell-membrane targets.

Li et $a l .{ }^{41}$ isolated 6 compounds from an extract of Danggui Buxue decoction that selectively combined with endothelial cells. Of these, Angelica sinensis Radix contributed 2, and Astragali Radix 4. Four of the 6 potentially bioactive compounds were elucidated using HPLC/MS and identified as ononoside, calycosin, 3-butylphthalide, and ligustilide. Fan et al. ${ }^{42}$ found 8 compounds in a sample of Mailuoning injection that may bind with platelet membrane, using centrifugal sedimentation. Zhang et al. $^{\mathbf{4 3}}$ found 14 active compounds from a glucose injection of zedoary turmeric oil that bound with living hepatocytes, using centrifugal sedimentation with GC/MS analysis. Of these, curzerene and $\beta$-elemene, which were of the highest content, were the main active ingredients. Zhang et al. ${ }^{\mathbf{4 4}}$ identified 9, 7 and 13 active compounds that could bind with HL7702, RAW 264.7 and Caco-2 cells, respectively, from an extract of Danggui Buxue decoction, using centrifugal sedimentation with LC/MS analysis. Using the same method, many active constituents binding different cells or cell membranes have been isolated from TCM extracts such as Scrophulariae Radix, Notoginseng Radix, Astragali Radix, Angelicae sinensis Radix, Zingiberis Rhizoma, Cordyceps, Artemisiae scopariae Herba, Epimedii Folium and Yu-ping-feng powder. ${ }^{20}$

3.5.2. Dialysis. A semi-permeable membrane is employed in dialysis to detain suspended solids and solutes of high molecular weight, whereas liquid and low-molecular-weight solutes are allowed to freely permeate the membrane, depending on the nominal molecular weight cut-off of the membrane. Using dialysis technology, screening targets binding with active constituents are entrapped by the semi-permeable membrane, while inactive constituents not bound to targets will permeate, separating active and inactive constituents. Concentrations of active compounds change remarkably when comparing concentrations (or contents) of compounds in TCM extract that are directly dialyzed, and those incubated with screening target followed by dialysis. Lei et $a .^{45}$ found that several active constituents bound with MCF-7 and multidrug resistant MCF-7 cells from the cortex of Pseudolarix kaempferi (Lamb.) Gordon and the root of Stephania tetrandrae S. Moore, respectively, using microdialysis sampling combined with HPLC/MS. Qi et al. $^{46}$ detected 15 active compounds binding with liposome from Danggui Buxue decoction, using dialysis with HPLC 
analysis. Dong et $a .^{47}$ identified 8 active compounds binding with liposomes from the water extract of Salviae miltiorrhizae Radix, using dialysis with HPLC/MS analysis. This method can be combined online with HPLC (or LC/MS) to assay active substances, and concentration changes of active compounds can be monitored in real time. However, this technology is unavailable for sample enrichment, resulting in low sensitivity, so it is thus unsuitable for measuring trace samples.

3.5.3. Magnetic separation. With magnetic separation, active constituents are captured by magnetic beads covalently bonded with screening targets and separated from complex mixtures using a magnetic field. The operational processes are as follows (Fig. 2): (1) the target protein is covalently coupled on magnetic beads to obtain "target protein-coupled magnetic beads" (TPCMB) through a condensation reaction between amino and carboxyl groups of the protein and the bead surface; (2) the analytical sample is incubated with TPCMB in an Eppendorf tube to allow binding of active constituents to targets; (3) the TPCMB bound to active constituents accumulate on the tube wall by applying a magnetic field to the outside of the tube; (4) supernatant containing inactive ingredients is removed, and active constituents coupled with TPCMB are dissociated with an organic solvent; (5) the TPСMB is discarded using the magnetic field, and the supernatant containing the active constituents is analyzed.

A variety of TPCMBs for different target proteins have been fabricated to screen for active constituents in complex matrices such as TCMs, and several active compounds have been found. Target proteins used have included human serum albumin, ${ }^{\mathbf{4 8 - 5 1}}$ bovine serum albumin, ${ }^{52}$ hVDR protein ${ }^{53}$ anti-streptavidin, ${ }^{54}$ protein tyrosine phosphatase $1 \mathrm{~B},{ }^{55} \alpha$-glucosidase, ${ }^{55}$ maltase, ${ }^{56}$ sucrase, ${ }^{56}$ lipases, ${ }^{56}$ estrogen receptor, ${ }^{57}$ SIRT6 protein, ${ }^{58}$ acetylcholinesterase,${ }^{59}$ heat shock protein, ${ }^{60}$ and calmodulin. ${ }^{61}$ Qing et al. identified 3 and 4 active compounds from extracts of Dioscorea nipponica ${ }^{\mathbf{4 8}}$ and Dioscorea panthaica, ${ }^{\mathbf{4 9}}$ respectively, using human serum albumin-coupled magnetic beads. Zhang et $a l .{ }^{50}$ used human serum albumin-coupled magnetic beads to isolate 47 active substances from Astragali Radix. Liu et al. ${ }^{52}$ prepared bovine serum albumin-coupled magnetic beads to extract 13 active compounds from Puerariae lobatae Radix. Arai et $a l .{ }^{53}$ prepared hVDR protein-coupled magnetic beads and found 2 active substances (ent-12(E)-labda-8(17),12,14-trien-18ol and ent-12(Z)-labda-8(17),12,14-trien-18-ol) in the ethanol extract of Eleutherine palmifolia. Qing et al. ${ }^{55}$ prepared protein tyrosine phosphatase 1B- and $\alpha$-glucosidase-coupled magnetic beads, respectively extracting 4 and 2 active compounds from the bark of Punica granatum L. Tao et al. ${ }^{56}$ developed a multitarget screening method connecting maltose-, sucrose- and lipase-coupled magnetic beads, and identified 5 active compounds inhibiting the 3 enzymes immobilized on magnetic beads from the TCM formula of Sanye Tangzhiqing. Yasuda et al. ${ }^{58}$ isolated 2 active compounds from an extract of common Fenugreek seed using prepared SIRT6 protein-coupled magnetic beads.

Although these results suggest that screening active constituents from complex matrices of TCMs can be achieved using TPCMB with magnetic field separation, the target protein may be denatured or its three-dimensional configuration may be changed when the target protein is coupled to magnetic beads. This results in mild binding with active compounds, or even unbinding. Additionally, coupling conditions should be optimized to maximally immobilize protein, which improves screening sensitivity.

3.5.4. Hollow fiber adsorption. During hollow fiber adsorption, screening targets are immobilized on the inner wall of a hollow fiber via physical adsorption. The operational processes are as follows: (1) the hollow fiber is immersed in the target solution, and the target directly adsorbed to the inner walls of the hollow fibers; (2) the hollow fibers adsorbed with targets are placed in the analytical sample solution to adsorb active molecules; (3) the fiber is removed from the solution, and washed to remove small molecules that have non-specifically adsorbed; (4) active substances are released from screening targets by denaturing with organic solvent, and then actives are assayed.

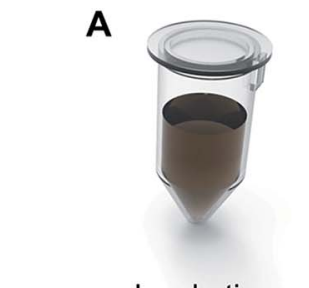

Incubation

Extract for screening + TPCMB
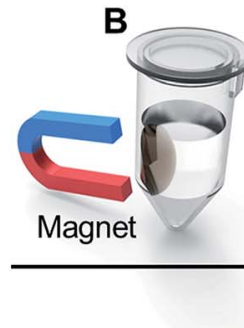

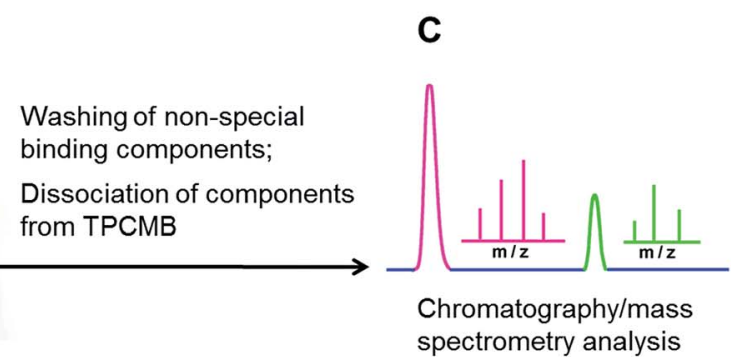

D

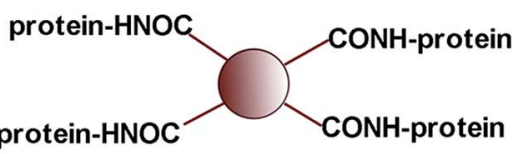

TPCMB

Fig. 2 The analytical process of magnetic separation for TCM bioactive component screening. (A) Incubation of the analyzed sample and TPCMB; (B) washing of non-special binding components and dissociation of bioactive components binding with target, using a magnetic field outside a tubal wall; (C) analysis of the dissociated bioactive components by chromatography/mass spectrometry; (D) TPCMB diagram. 
Hollow fiber adsorption has been used to screen living cell, cell membranes, organelles, and enzyme targets. Xue et al. ${ }^{\mathbf{6 2}}$ identified a diverse active compounds from extracts of Schisandrae chinensis Fructus, Cnidii Fructus and Psoraleae Fructus using hollow fiber adsorbed with living cells. Liu et al. ${ }^{63}$ isolated several active compounds from Coptidis Rhizoma using 3 types of hollow fiber, respectively adsorbed with 3 different targets (MCF-7 cells, cell membranes and organelles). Yan et al. ${ }^{\mathbf{6 4}}$ found several anthraquinone compounds that could penetrate cells from extracts of Polygoni cuspidati Rhizoma, Cassiae obtusifoliae Semen and Polygoni multiflori Radix. Tao et al. ${ }^{65}$ isolated 3 lipase-bound ligands (quercetin-3-O- $\beta$-D-arabinopyranosyl- $(1 \rightarrow$ $2)$ - $\beta$-D-galactopyranoside, quercetin-3-O- $\beta$-D-glucuronide and kaempferol-3-O- $\beta$-D-glucuronide) from extracts of lotus leaf, using hollow fiber adsorbed with lipases from porcine pancreas. Despite these successes, limitations persist. The target adsorbed on the inner wall of the hollow fiber has a short survival time and only few targets are adsorbed, restricting the sensitivity of this method.

3.5.5. Affinity ultrafiltration. Affinity ultrafiltration employs affinity capture and ultrafiltration, enabling the enrichment and separation of low-molecular-weight solutes bound to biomacromolecules (such as enzymes, receptors, carrier proteins and DNA). Suspended solids and solutes of high molecular weight are retained by the semi-permeable ultrafiltration membrane depending on the nominal molecular weight cut-off of the membrane. ${ }^{66}$ This is depicted in Fig. $3:^{67-69}$ (1) the analyzed sample is incubated with the screening target which facilitates binding to target; (2) the inactive molecules non-specifically absorbed by the target are washed with buffer and removed by ultrafiltration; (3) the target is deactivated using addition of organic solvent or $\mathrm{pH}$ alteration, which contributes to the dissociation of active molecules from the target. Active disassociated molecules are then separated from the target solution via ultrafiltration; (4) the ultrafiltrate containing active molecules is analyzed with HPLC, MS, or HPLC/MS.
Compared with other active molecular screening methods such as biochromatography, magnetic separation, and hollow fiber adsorption, affinity ultrafiltration has significant advantages: ${ }^{70}$ (1) it is not necessary to immobilize the screening targets on a chromatographic carrier. The target is directly incubated with the samples, which maintain their native conformation and avoid a reduction in activity, deactivation, or conformational change originating from the target immobilization; (2) pulse ultrafiltration can be conveniently coupled with online MS (or with a fraction trap, followed by online LC/MS), for online analysis of active constituents; (3) affinity ultrafiltration is convenient to operate, low-cost, and has a high hit ratio.

Many active molecules have been found in a variety of natural products and combinatorial libraries using affinity ultrafiltration combined with MS or LC/MS (Table 1), suggesting that affinity ultrafiltration could be used for screening active constituents from products with complex matrices, such as TCMs.

\subsection{Bioreactor}

In a bioreactor, analytical samples are incubated with screening targets, and reaction substrates are added to initiate a biochemical reaction under enzymatic catalysis. The concentrations (or contents) of active compounds change during this process and reactions can be divided into pre-, in- and aftercolumn according to the installation site of the bioreactor.

With a pre-column reaction, reacted samples are separated and measured with chromatography (or electrophoresis), which is convenient and sensitive. Mou et al. ${ }^{103}$ cultured LoVo cells on a porous PHC scaffold to develop a three-dimensional cell bioreactor. Combined with HPLC/MS, this bioreactor was used to screen for active constituents in Polygonum cillinerve (Nakai) Ohwi extract, resulting in the identification of 2 active compounds (aristolochic acid A and aristolochic acid B).

With an in-column reaction, the screening targets (enzymes) are immobilized on a support and then packed in a column.

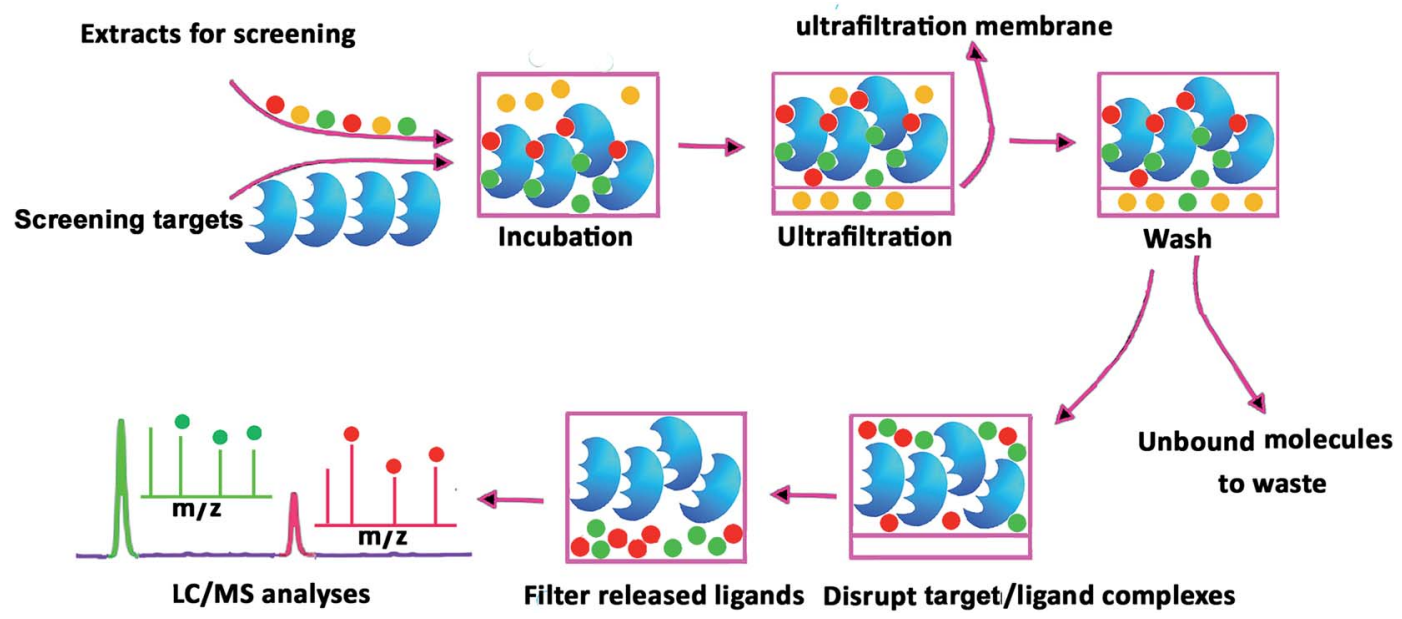

Fig. 3 Analytical procedure for the identification of bioactive constituents from complex mixtures, using centrifugal ultrafiltration/liquid chromatography/mass spectrometry method. 
Table 1 Natural products and combinatorial libraries screened by affinity ultrafiltration

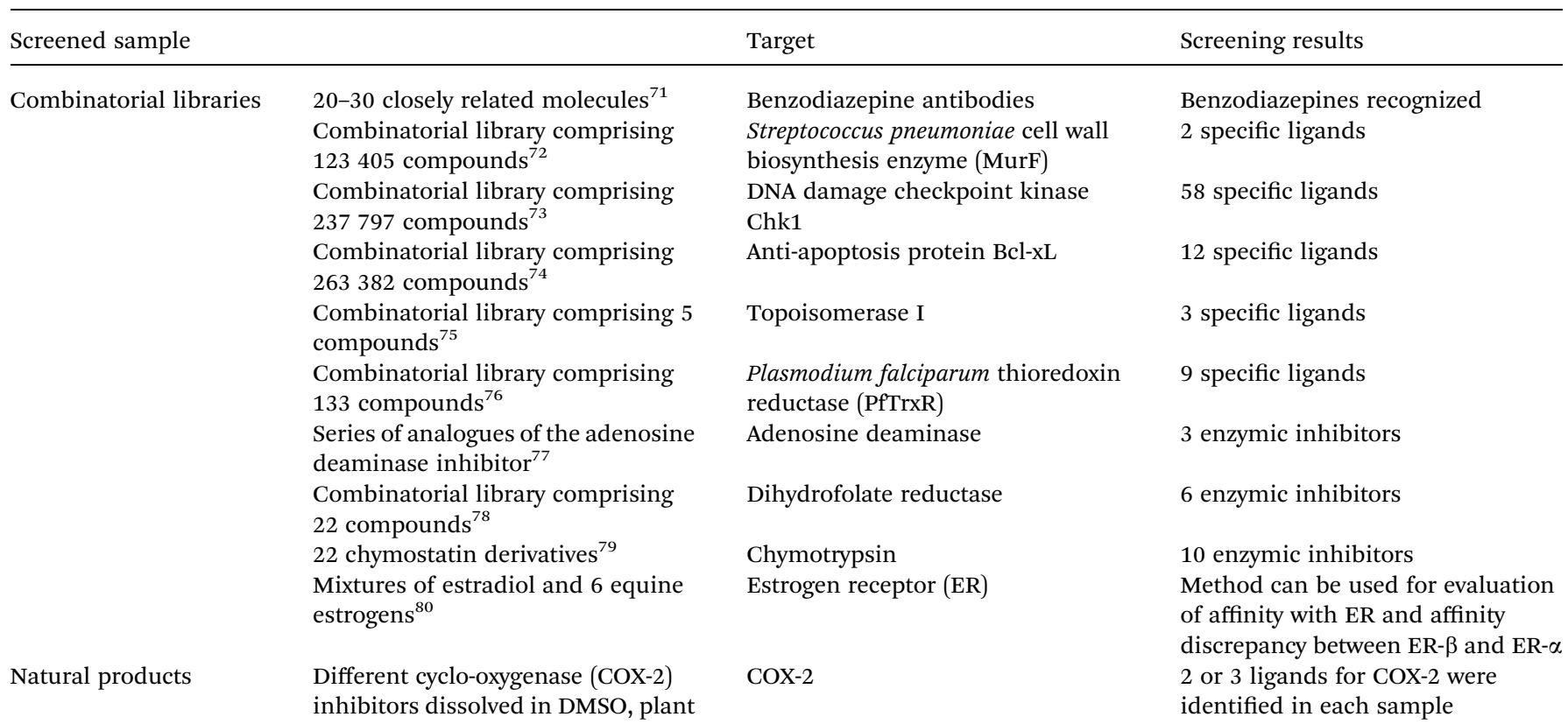

extract and bacterial fermentation broth extract ${ }^{81}$

Methanolic fermentation broth extract spiked with known inhibitor to carbonic anhydrase ${ }^{82}$

TCM Huo-Luo-Xiao-Ling Dan ${ }^{83}$

Botanical extracts and their metabolites obtained by hepatic cytochrome P450 (ref. 84) Lonicerae japonicae Flos extract ${ }^{85}$ Extract of a marine sediment bacterial culture ${ }^{86}$

Cells grown in culture ${ }^{64}$

Mulberry leaf extracts ${ }^{87}$

Scutellaria Radix extract ${ }^{88}$

Salviae miltiorrhizae Radix extract ${ }^{89}$

Water extract of Eucommia ulmoides bark $^{90}$

Extract of marine sediment bacteria $^{91}$

Extract of Humulus lupulus L. $^{91}$ Water extract of Eucommia ulmodies leaves $^{92}$

Lonicerae japonicae Flos extract ${ }^{93}$

Extract of Lonicera japonica

Thunb. ${ }^{94}$

TCM red clover isoflavone extracts ${ }^{95}$

Extracts of Macleaya cordata,

Chelidonium majus and Coptis chinensis $^{96}$

Hawthorn leaf flavonoids extract ${ }^{97}$ Extract of leaves of Acanthopanax senticosus harms ${ }^{98}$

Total saponins of ginseng stems and leaves ${ }^{99}$

Extract of Polygonatum odoratum ${ }^{100}$ Astragali Radix extract and its human microsomal metabolites ${ }^{101}$

Carbonic anhydrase and serum albumin

Cyclo-oxygenase

Glutathione (GSH)

Liposome

Human retinoid X receptors (RXRs)

Topoisomerase I

Tyrosinase

Neuraminidase

Xanthine oxidase

Phosphodiesterase

Quinine reductase-2

Quinine reductase-2

Bovine serum albumin

Bovine serum albumin

DNA

DNA

DNA

$\alpha$-Glucosidase

$\alpha$-Glucosidase

$\alpha$-Glucosidase

$\alpha$-Glucosidase

$\alpha$-Glucosidase

Mitochondria
Non-covalent ligand binding to both targets observed

17 specific ligands Several compounds that formed GSH conjugates

8 permeable compounds

2 specific ligands

1 enzymic inhibitor

2 enzymic inhibitors

6 enzymic inhibitors

11 enzymic inhibitors

7 enzymic inhibitors

1 enzymic inhibitor

2 enzymic inhibitors

6 special ligands

11 special ligands

4 DNA binders

5 DNA binders

9 DNA binders

4 enzymic inhibitors

8 enzymic inhibitors

12 enzymic inhibitors

9 enzymic inhibitors

13 prototype isoflavonoids and 1 monohydroxylated metabolic isoflavonoid with enzymic binding activity

19 active compounds detected and identified 
Samples are injected into the column and incubated with the enzymes and substrates. After the biochemical reaction, reactive samples are separated using electrophoresis (or chromatography). This method is highly stable and repeatable, with little enzyme consumption, but the immobilized enzymes may be denatured or inactivated. L-Glutamate dehydrogenases were immobilized on the surface of gold nanoparticles, and then packed into a capillary column to develop an online enzyme microreactor, by Zhao et al. ${ }^{\mathbf{1 0 4}}$ Using this microreactor, 3 TCM extracts that had inhibitory activity were identified from 25 extracts. Ji et al. ${ }^{\mathbf{1 0 5}}$ prepared a microreactor of adenosine deaminase and identified one inhibitory extract from 19 TCM extracts.

In an after-column reaction, the analytical samples are separated by chromatography (electrophoresis), and separated fractions are reacted with screening targets (enzymes). Changes in sample components are then measured. Interference within the biochemical reaction from matrices and other components of complex samples is less with this method. However, online sample detection is difficult and special equipment and techniques are required. Li et al. ${ }^{\mathbf{1 0 6}}$ developed online microreactors for xanthine oxidase and ABTS radical cation $\left(\mathrm{ABTS}^{*}\right)$ to screen for xanthine oxidase inhibitors and free radical scavengers, respectively, from Oroxyli Semen extract. Six free radical scavengers, a xanthine oxidase inhibitor and a compound with free radical scavenging and xanthine oxidase inhibiting activity were identified. Lin et al. ${ }^{\mathbf{1 0 7}}$ next prepared luminol and ferricyanide microreactors to screen Aurantii Fructus for antioxidants and found 25 active compounds.

\subsection{Gene chip technology}

Genes carry genetic information and drugs act through different targets, directly or indirectly affecting cellular gene expression. DNA microarray gene expression profiling is the gene chip generally used for drug screening. ${ }^{108}$ By comparing genetic expression of two samples before and after drug administration, changes in expression may help to identify the target gene of the drug. Gene chips for drug screening is low with respect to labor intensity and animal consumption and can be carried out with small samples. Gene chip screening also has a short cycle, which decreases time and cost of drug screening, so it is popular for screening active constituents in TCMs. ${ }^{\text {109-113 }}$ Gene chip analysis can be improved by an increase in chip specificity, simplification of sample preparation and marking, improvements in signal detection sensitivity and stability, and additional cost reduction.

\subsection{Molecularly imprinted polymers}

Molecular imprinting is used to prepare polymers containing recognition sites of predetermined specificity. Molecularly imprinted polymers (MIPs) are complexed in a solution of template molecules with functional monomers by covalent or non-covalent bonds, followed by a polymerization reaction with an excess of cross-linkers. Removal of the templates leaves specific recognition sites that are complementary to the template in terms of its shape, size and functionality in the polymer network. ${ }^{\mathbf{1 1 4}}$ The perfect selectivity, high binding affinity and physical robustness of MIPs allow their application for nonbiological screening in lead compound discovery. Structures of affinitive compounds, which were trapped by MIPs from the mixture, were similar to the template and should have similar bioactivity according to structure-activity relationships. ${ }^{115}$

Despite MIPs require $\sim 1 \mathrm{mmol}$ of pure template molecule and have difficulty dealing with macromolecules such as proteins and polynucleotides, they have been used for screening analogues with similar bioactivity in the hope of finding new drug candidates from plant extracts or combinatorial chemistry libraries. Yang's group ${ }^{\mathbf{1 1 5}}$ prepared oseltamivir MIPs and identified an active component against influenza virus (matrine) from chloroform extracts of a TCM formula of Yandureqing. Huang and colleagues $^{\mathbf{1 1 6}}$ prepared propyl gallate MIPs and identified an antiplatelet active ingredient (protocatechuic acid) from Salviae miltiorrhizae Radix. Furthermore, (-)-ephedrine-, (E)-piceatannol-, fisetin-, harman- and RD3-4078-imprinted polymers were prepared for screening and separating active natural compounds from TCMs. ${ }^{114}$ Steroid receptor, folate receptor and $\alpha 2$-adrenoreceptor mimics have been made by MIPs and used to screen bioactive compounds from synthetic libraries. ${ }^{\mathbf{1 1 4}}$

\section{Spectrum-effect relationship}

Chemical constituents and pharmacological studies have been unable to clarify how TCM substances can treat particular diseases. Exploring the spectrum-effect relationship (SER) by combining chemical constituent studies with pharmacological studies may clarify overall therapeutic effects and the relationship between active constituents. SER is widely applied in research into the basis for substance effectiveness, compatibility, and the processing of TCMs ${ }^{117,118}$ as follows: (1) fingerprinting of TCM samples from different environments, manufacturers or with different preparation methods is developed to profile chemical discrepancies across different samples; (2) difference in sample efficacy is evaluated by in vivo or in vitro experiments; (3) statistical analysis, such as principal components analysis, direct-vision comparison, and gray relational analysis, is performed to establish the SER, revealing the most effective TCM substances. Xu et al. ${ }^{118}$ reviewed the current status of and future perspectives in SER for TCM research and various active constituents in single TCMs and TCM formulas have been identified. Thus, SER analysis may potentially determine the effective substances in complex mixtures and reflect the internal quality of TCMs.

Although SER research has been used successfully, optimization is needed: (1) TCM fingerprinting may have poor stability and resolution; (2) modern pharmacological models that are currently established within western medicine theory may not adequately reflect the clinical efficacy of TCMs; (3) important factors affecting the stability and reliability of SER research results are not investigated systematically; (4) uniform standards for quantitative indicators of efficacy are lacking; (5) the relationships between the active constituents in TCMs are not clear. In summary, the application of SERs in TCM research needs further exploration. 


\section{Conclusion}

Identifying lead compounds from natural products plays a vital role in the search for successful drug candidates. Furthermore, clarifying the effective substance basis of TCMs is a prerequisite for recognizing TCM efficacy and ensuring TCM quality. However, the complex chemical composition and action mechanisms of TCMs mean that effective substance research is a slow process. Various methods and techniques have been applied to the exploration of the effective substances in commonly used TCMs that treat certain disorders, with valuable results. However, research methods continue to have limitations. New research ideas, and the further development of screening techniques to improve the efficiency and effectiveness of screening for the active constituents of TCMs, are required. This paper's documentation of the techniques currently used may assist readers with the rapid retrieval of useful information, and meaningfully promote the continued search for an effective substance basis to TCM, improving quality control and modernization.

\section{Acknowledgements}

Financial support from the National Natural Science Foundation of China (Grant 81660596 and 81460623), the Application and Basis Research Project of Yunnan China (Grant 2016FD050), the Natural Science Foundation of Yunnan China (Grant 2014FA035), and the Young and Middle-aged Academic and Technological Leader of Yunnan (Grant 2015HB053) is gratefully acknowledged.

\section{References}

1 K. Chen, Trends Pharmacol. Sci., 1995, 16, 182-187.

2 D. Normile, Science, 2003, 299, 188-190.

3 R. Yuan and Y. Lin, Pharmacol. Ther., 2000, 86, 191-198.

4 J. F. Wang, D. Q. Wei and K. C. Chou, Curr. Top. Med. Chem., 2008, 8, 1656-1665.

5 Y. Wang, X. Fan, H. Qu, X. Gao and Y. Cheng, Curr. Top. Med. Chem., 2012, 12, 1356-1362.

6 B. C. Wang, J. Deng, Y. M. Gao, L. C. Zhu, R. He and Y. Q. Xu, Fitoterapia, 2011, 82, 1141-1151.

7 S. M. Chan and J. M. Ye, J. Pharm. Pharm. Sci., 2013, 16, 207216.

8 X. Huang, X. L. Kong, X. Chen, M. Guo and H. F. Zou, J. Chromatogr. B: Anal. Technol. Biomed. Life Sci., 2004, 812, 71-84.

9 X. J. Wang, World J. Sci. Technol., 2002, 4, 1-4.

10 G. L. Yan, H. Sun, A. H. Zhang, Y. Han, P. Wang, X. H. Wu, X. C. Meng and X. J. Wang, Zhongguo Zhongyao Zazhi, 2015, 40, 3406-3412.

11 F. Gao, Y. Hu, G. Fang, G. Yang, Z. Xu, L. Dou, Z. Chen and G. Fan, J. Pharm. Biomed. Anal., 2014, 87, 241-260.

12 O. P. Kallioniemi, J. Kononen and G. Sauter, Clin. Chem., 2012, 58, 1717-1718.

13 J. Z. Pan, R. Jörnsten and R. P. Hart, Physiol. Genomics, 2004, 17, 201-214.
14 F. Xu, J. Wu and S. Wang, Biofabrication, 2011, 3, 034101. 15 K. Tsuganezawa, Y. Nakagawa, M. Taruya, S. F. Takahashi, M. Endoh, R. Utata, M. Mori, N. Ogawa, T. Honma, S. Yokoyama, Y. Hashizume, M. Aoki, T. Kasai, T. Kigawa, H. Kojima, T. Okabe, T. Nagano and A. Tanaka, J. Biomol. Screening, 2013, 18, 191-198.

16 M. V. Tsiper, J. Sturgis, L. V. Avramova, S. Parakh, R. Fatig, A. Juan-García, N. Li, B. Rajwa, P. Narayanan, C. W. Qualls, J. P. Robinson and V. J. Davisson, PLoS One, 2012, 7, e45226.

17 L. Tolosa, M. J. Gómez-Lechón, G. Pérez-Cataldo, J. V. Castell and M. T. Donato, Arch. Toxicol., 2013, 87, 1115-1127.

18 Y. Shen, Y. Hu, B. Chen and S. Yao, Comb. Chem. High Throughput Screening, 2010, 13, 885-899.

19 Y. Zhu, Z. Zhang, M. Zhang, D. E. Mais and M. W. Wang, Comb. Chem. High Throughput Screening, 2010, 13, 837-848.

20 C. Chen, F. Q. Yang, H. L. Zuo, Y. L. Song, Z. N. Xia and W. Xiao, J. Chromatogr. Sci., 2013, 51, 780-790.

21 F. Yu, L. Kong, H. Zou and X. Lei, Comb. Chem. High Throughput Screening, 2010, 13, 855-868.

22 X. X. Yang, Y. L. Zhang, X. X. Zhang and X. N. Li, J. Chin. Pharm. Sci., 2011, 20, 20-25.

23 X. F. Hou, M. Z. Zhou, Q. Jiang, S. C. Wang and L. C. He, J. Chromatogr. A, 2009, 1216, 7081-7087.

24 S. C. Wang, M. Sun, Y. M. Zhang, H. Du and L. C. He, J. Chromatogr. A, 2010, 1217, 5246-5252.

25 X. X. Yang, Y. W. Wang, X. X. Zhang, R. M. Chang and X. N. Li, J. Sep. Sci., 2011, 34, 2586-2593.

26 Y. Wang, L. Kong, L. Hu, X. Lei, L. Yang, G. Chou, H. Zou, C. Wang, S. W. Annie Bligh and Z. Wang, J. Chromatogr. B: Anal. Technol. Biomed. Life Sci., 2007, 860, 185-194.

27 S. Wang, C. Wang, X. Zhao, S. Mao, Y. Wu and G. Fan, Anal. Chim. Acta, 2012, 713, 121-129.

28 Y. Wang, L. Kong, X. Lei, L. Hu, H. Zou, E. Welbeck, S. W. Bligh and Z. Wang, J. Chromatogr. A, 2009, 1216, 2185-2191.

29 D. Jia, X. Chen, Y. Cao, X. Wu, X. Ding, H. Zhang, C. Zhang, Y. Chai and Z. Zhu, J. Pharm. Biomed. Anal., 2016, 118, 2733.

30 X. Su, L. Kong, X. Lei, L. Hu, M. Ye and H. Zou, Mini-Rev. Med. Chem., 2007, 7, 87-98.

$31 \mathrm{H}$. Wang, L. Kong, H. Zou, J. Ni and Y. Zhang, Chromatographia, 1999, 50, 439-445.

32 X. Su, L. Hu, L. Kong, X. Lei and H. Zou, J. Chromatogr. A, 2007, 1154, 132-137.

33 W. A. Min, W. P. Wang, J. R. Chen, A. Wang and Z. Hu, Anal. Bioanal. Chem., 2012, 404, 2397-2405.

34 X. Hou, S. Wang, T. Zhang, J. Ma, J. Zhang, Y. Zhang, W. Lu, H. He and L. He, J. Pharm. Biomed. Anal., 2014, 101, 141150.

35 S. Muhammad, S. Han, X. Xie, S. Wang and M. M. Aziz, J. Sep. Sci., 2016, 1-15.

36 R. L. Pignatello, T. Musumeci, L. Basile, C. Carbone and G. Puglisi, J. Pharm. BioAllied Sci., 2011, 3, 4-14.

37 C. Zhang, J. Li, L. Xu and Z. G. Shi, J. Chromatogr. A, 2012, 1233, 78-84. 
38 C. Zeng, Y. Zhang, L. Lu, E. Brekkan, A. Lundqvist and P. Lundahl, Biochim. Biophys. Acta, 1997, 1325, 91-98.

39 R. Ehwald, H. Woehlecke and C. Titel, Phytochemistry, 1992, 31, 3033-3038.

40 G. Dongow ski, R. Ehwald, K. Luck and G. Stoof, Phytochemistry, 1992, 31, 3039-3042.

41 S. L. Li, P. Li, L. H. Sheng, R. Y. Li, L. W. Qi and L. Y. Zhang, J. Pharm. Biomed. Anal., 2006, 41, 576-581.

42 H. W. Fan, Q. Zhu, M. Hong and L. Yu, Chin. Pharm. J., 2006, 41, 63-66.

43 H. Y. Zhang, C. X. Hu, C. P. Liu, H. F. Li, J. S. Wang, K. L. Yuan, J. W. Tang and G. W. Xu, J. Pharm. Biomed. Anal., 2007, 43, 151-157.

44 X. Zhang, L. W. Qi, L. Yi, P. Li, X. D. Wen and Q. T. Yu, Biomed. Chromatogr., 2008, 22, 157-163.

45 X. Lei, L. Kong, H. Zou, H. Ma and L. Yang, J. Chromatogr. A, 2009, 1216, 2179-2184.

46 L. W. Qi, P. Li and L. H. Sheng, Chin. J. Anal. Chem., 2006, 34, 196-199.

47 Q. Q. Dong, R. Y. Li and P. Li, Chin. Pharm. J., 2007, 42, 1208-1211.

48 L. S. Qing, Y. Xue, Y. Zheng, J. Xiong, X. Liao, L. S. Ding, B. G. Li and Y. M. Liu, J. Chromatogr. A, 2010, 1217, 46634668.

49 L. S. Qing, X. Q. Shan, X. M. Xu, Y. Xue, W. L. Deng, B. G. Li, X. L. Wang and X. Liao, Rapid Commun. Mass Spectrom., 2010, 24, 3335-3339.

50 Y. Zhang, M. Nie, S. Shi, Q. You, J. Guo and L. Liu, Food Chem., 2014, 146, 56-64.

51 L. S. Qing, Y. Xue, W. L. Deng, X. Liao, X. M. Xu, B. G. Li and Y. M. Liu, Anal. Bioanal. Chem., 2011, 399, 1223-1231.

52 L. Liu, Y. Ma, X. Chen, X. Xiong and S. Shi, J. Chromatogr. B: Anal. Technol. Biomed. Life Sci., 2012, 887-888, 55-60.

53 M. A. Arai, E. Kobatake, T. Koyano, T. Kowithayakorn, S. Kato and M. Ishibashi, Chem.-Asian J., 2009, 4, 18021808.

54 X. L. Yang, J. Pu, H. Zhao, X. Y. Li, J. Liao, Y. L. Xie, S. Zhu, G. B. Long, Y. H. Yuan and F. Liao, Microchim. Acta, 2012, 176, 243-249.

55 L. S. Qing, N. Tang, Y. Xue, J. Liang, Y. M. Liu and X. Liao, Anal. Methods, 2012, 4, 1612-1615.

56 Y. Tao, Z. Chen, Y. Zhang, Y. Wang and Y. Cheng, J. Pharm. Biomed. Anal., 2013, 78-79, 190-201.

57 Y. Choi and R. B. van Breemen, Comb. Chem. High Throughput Screening, 2008, 11, 1-6.

58 M. Yasuda, D. R. Wilson, S. D. Fugmann and R. Moaddel, Anal. Chem., 2011, 83, 7400-7407.

59 L. Pochet, F. Heus, N. Jonker, H. Lingeman, A. B. Smit, W. M. Niessen and J. Kool, J. Chromatogr. B: Anal. Technol. Biomed. Life Sci., 2011, 879, 1781-1788.

60 M. P. Marszall, R. Moaddel, S. Kole, M. Gandhari, M. Bernier and I. W. Wainer, Anal. Chem., 2008, 80, 75717575.

61 M. J. McFadden, M. S. Junop and J. D. Brennan, Anal. Chem., 2010, 82, 9850-9857.

62 X. Xue, L. Li, X. Chen, S. Hu and X. Bai, J. Chromatogr. A, 2013, 1280, 75-83.
63 X. Liu, S. Hu, X. Chen and X. Bai, J. Pharm. Biomed. Anal., 2014, 98, 463-475.

64 Y. Yan, Y. Hao, S. Hu, X. Chen and X. Bai, J. Chromatogr. A, 2013, 1322, 8-17.

65 Y. Tao, Y. Zhang, Y. Wang and Y. Cheng, Anal. Chim. Acta, 2013, 785, 75-81.

66 D. A. Annis, E. Nickbarg, X. Yang, M. R. Ziebell and C. E. Whitehurst, Curr. Opin. Chem. Biol., 2007, 11, 518-526.

67 K. M. Comess and M. E. Schurdak, Curr. Opin. Drug Discovery Dev., 2004, 7, 411-416.

68 T. E. Cloutier, Library screening using ultrafiltration and mass spectrometry, Wiley-VCH, Weinheim, 2007, pp. 157-184.

69 D. Liu, J. Guo, Y. Luo, D. J. Broderick, M. I. Schimerlik, J. M. Pezzuto and R. B. van Breemen, Anal. Chem., 2007, 79, 9398-9402.

70 B. M. Johnson, D. Nikolic and R. B. van Breemen, Mass Spectrom. Rev., 2002, 21, 76-86.

71 R. Wieboldt, J. Zweigenbaum and J. Henion, Anal. Chem., 1997, 69, 1683-1691.

72 K. M. Comess, M. E. Schurdak, M. J. Voorbach, M. Coen, J. D. Trumbull, H. Yang, L. Gao, H. Tang, X. Cheng, C. G. Lerner, J. O. McCall, D. J. Burns and B. A. Beutel, J. Biomol. Screening, 2006, 11, 743-754.

73 K. M. Comess, J. D. Trumbull, C. Park, Z. Chen, R. A. Judge, M. J. Voorbach, M. Coen, L. Gao, H. Tang, P. Kovar, X. Cheng, M. E. Schurdak, H. Zhang, T. Sowin and D. J. Burns, J. Biomol. Screening, 2006, 11, 755-764.

74 J. Qian, M. J. Voorbach, J. R. Huth, M. L. Coen, H. Zhang, S. C. Ng, K. M. Comess, A. M. Petros, S. H. Rosenberg, U. Warrior and D. J. Burns, Anal. Biochem., 2004, 328, 131-138.

75 H. Zhang, Q. Gu, X. Liang and Y. Pan, Anal. Biochem., 2004, 329, 173-179.

76 R. Munigunti, V. Mulabagal and A. I. Calderón, J. Pharm. Biomed. Anal., 2011, 55, 265-271.

77 Y. Z. Zhao, R. B. Van Breemen, D. Nikolic, C. R. Huang, C. P. Woodbury, A. Schilling and D. L. Venton, J. Med. Chem., 1997, 40, 4006-4012.

78 D. Nikolic and R. B. Van Breemen, Comb. Chem. High Throughput Screening, 1998, 1, 47-55.

79 S. Mathur, M. Hassel, F. Steiner, K. Hollemeyer and R. W. Hartmann, J. Biomol. Screening, 2003, 8, 136-148.

80 Y. Sun, C. Gu, X. Liu, W. Liang, P. Yao, J. L. Bolton and R. B. van Breemen, J. Am. Soc. Mass Spectrom., 2005, 16, 271-279.

81 D. Nikolic, S. Habibi-Goudarzi, D. G. Corley, S. Gafner, J. M. Pezzuto and R. B. van Breemen, Anal. Chem., 2000, 72, 3853-3859.

82 M. B. Beverly, P. West and R. K. Julian, Comb. Chem. High Throughput Screening, 2002, 5, 65-73.

83 H. M. Cao, R. Yu, Y. S. Choi, Z. Z. Ma, H. Zhang, W. Xiang, D. Y. Lee, B. M. Berman, K. D. Moudgil, H. H. Fong and R. B. van Breemen, Pharmacol. Res., 2010, 61, 519-524.

84 B. M. Johnson, J. L. Bolton and R. B. van Breemen, Chem. Res. Toxicol., 2001, 14, 1546-1551.

85 X. P. Chen, Y. Q. Xia, Y. Lu and J. Liang, J. Pharm. Biomed. Anal., 2011, 54, 406-410. 
86 D. Liu, J. Guo, Y. Luo, D. J. Broderick, M. I. Schimerlik, J. M. Pezzuto and R. B. van Breemen, Anal. Chem., 2007, 79, 9398-9402.

87 Z. Yang, Y. Zhang, L. Sun, Y. Wang, X. Gao and Y. Cheng, Anal. Chim. Acta, 2012, 719, 87-95.

88 S. Liu, J. P. Xing, J. Yan, F. R. Song, Z. Q. Liu and S. Y. Liu, Acta Chim. Sin., 2011, 69, 1570-1574.

89 Y. Liu, S. Liu and Z. Liu, J. Chromatogr. B: Anal. Technol. Biomed. Life Sci., 2013, 923-924, 48-53.

90 S. Y. Shi, M. J. Peng, Y. P. Zhang and S. Peng, Anal. Bioanal. Chem., 2013, 405, 4213-4223.

91 Y. Choi, K. Jermihov, S. J. Nam, M. Sturdy, K. Maloney, X. Qiu, L. R. Chadwick, M. Main, S. N. Chen, A. D. Mesecar, N. R. Farnsworth, G. F. Pauli, W. Fenical, J. M. Pezzuto and R. B. van Breemen, Anal. Chem., 2011, 83, 1048-1105.

92 Y. Zhang, M. Peng, L. Liu, S. Shi and S. Peng, J. Agric. Food Chem., 2012, 60, 3119-3125.

93 Z. M. Qian, S. J. Qin, L. Yi, H. J. Li, P. Li and X. D. Wen, Biomed. Chromatogr., 2008, 22, 202-206.

94 J. L. Zhou, Z. M. Qian, Y. D. Luo, D. Tang, H. Chen, L. Yi and P. Li, Biomed. Chromatogr., 2008, 22, 1164-1172.

95 L. Ma, Z. F. Wang, L. N. Chen, F. R. Song, Z. Q. Liu and S. Y. Liu, Chem. J. Chin. Univ., 2013, 34, 331-335.

96 Z. F. Zhang, Y. Guo and X. H. Wei, Zhongguo Zhongyao Zazhi, 2011, 36, 2684-2688.

97 H. L. Li, F. R. Song, J. P. Xing, R. Tsao, Z. Liu and S. Liu, J. Am. Soc. Mass Spectrom., 2009, 20, 1496-1503.

98 H. Zhou, J. Xing, S. Liu, F. Song, Z. Cai, Z. Pi, Z. Liu and S. Liu, Phytochem. Anal., 2012, 23, 315-323.

99 Z. M. He, X. H. Wang, G. F. Li, J. M. Sun, H. Yang, Y. G. Gao and L. K. Zhang, Chin. J. Anal. Chem., 2013, 41, 1694-1698.

100 X. Zhou, J. Liang, Y. Zhang, H. Zhao, Y. Guo and S. Shi, J. Chromatogr. B: Anal. Technol. Biomed. Life Sci., 2015, 985, 149-154.

101 H. Zhao, Y. Zhang, Y. Guo and S. Shi, J. Pharm. Biomed. Anal., 2015, 104, 31-37.
102 X. X. Yang, F. Xu, D. Wang, Z. W. Yang, H. R. Tan, M. Y. Shang, X. Wang and S. Q. Cai, J. Chromatogr. A, 2015, 1413, 33-46.

103 Z. L. Mou, X. N. Qi, R. L. Liu, J. Zhang and Z. Q. Zhang, J. Chromatogr. A, 2012, 1243, 33-38.

104 S. Zhao, X. Ji, P. Lin and Y. M. Liu, Anal. Biochem., 2011, 411, 88-93.

105 X. W. Ji, F. G. Ye, P. Li and S. L. Zhao, Talanta, 2010, 82, 1170-1174.

106 D. Q. Li, J. Zhao and S. P. Li, J. Chromatogr. A, 2014, 1345, 50-56.

107 Z. Lin, H. Wang, Y. Xu, J. Dong, Y. Hashi and S. Chen, Food Chem., 2012, 134, 1181-1191.

108 C. Debouck and P. N. Goodfellow, Nat. Genet., 1999, 21, 4850.

109 H. M. Cheng, C. C. Li, C. Y. Chen, H. Y. Lo, W. Y. Cheng, C. H. Lee, S. Z. Yang, S. L. Wu, C. Y. Hsiang and T. Y. Ho, J. Ethnopharmacol., 2010, 132, 429-437.

110 S. Katz, R. Harris, J. T. Y. Lau and A. Chau, J. Evidence-Based Complementary Altern. Med., 2006, 3, 65-70.

111 M. Yan, L. Y. Zhang, L. X. Sun, Z. Z. Jiang and X. H. Xiao, J. Ethnopharmacol., 2006, 107, 308-311.

112 A. Hara, N. lizuka, Y. Hamamoto, S. Uchimura, T. Miyamoto, R. Tsunedomi, K. Miyamoto, S. Hazama, K. Okita and M. Oka, Life Sci., 2005, 77, 991-1002.

113 N. Lizuka, M. Oka, K. Yamamoto, A. Tangoku, K. Miyamoto, T. Miyamoto, S. Uchimura, Y. Hamamoto and K. Okita, Int. J. Cancer, 2003, 107, 666-672.

114 X. J. Xu, Drug Discovery Today: Technol., 2006, 3, 247-253.

115 Y. J. Yang, J. Y. Li, X. W. Liu, J. Y. Zhang, Y. R. Liu and B. Li, PLoS One, 2013, 8, e84458.

116 M. Huang, W. Pang, J. Zhang, S. Lin and J. Hu, J. Pharm. Biomed. Anal., 2012, 58, 12-18.

117 C. S. Zhu, Z. J. Lin, M. L. Xiao, H. J. Niu and B. Zhang, Chin. J. Nat. Med., 2016, 14, 177-184.

118 G. L. Xu, M. Xie, X. Y. Yang, Y. Song, C. Yan, Y. Yang, X. Zhang, Z. Z. Liu, Y. X. Tian, Y. Wang, R. Jiang, W. R. Liu, X. H. Wang and G. M. She, Molecules, 2014, 19, 17897-17925. 